\title{
Feasibility of in vivo whole heart DTI and IVIM with a 15 minute acquisition protocol
}

\author{
Martijn Froeling ${ }^{1,2^{*}}$, Gustav J Strijkers ${ }^{2}$, Aart J Nederveen ${ }^{3}$, Steven A Chamuleau ${ }^{4}$, Peter R Luijten ${ }^{1}$ \\ From 17th Annual SCMR Scientific Sessions \\ New Orleans, LA, USA. 16-19 January 2014
}

\section{Background}

In recent years in vivo cardiac DTI using stimulated echo's (STE) has matured into a reproducible technique. However the STE approach requires two heartbeats and intrinsically has a $50 \%$ lower SNR compared to spin-echo (SE). Although the STE method allows for short TE $(23 \mathrm{~ms})$ it also suffers from T1 signal decay and typically 8 signal averages ( 16 heartbeats) are needed for a single slice acquisition. In this study we aimed to develop a SE-based cardiac diffusion MRI protocol that allows for whole heart DTI as well as intra-voxel coherent motion (IVIM) for perfusion assessment.

\section{Methods}

Images were acquired with cardiac triggering $(200 \mathrm{~ms})$ and free breathing on a 3T scanner (Philips, Achieva) using a 16-channel coil (Torso XL). DWI was performed using a SE sequence with bipolar diffusion weighting gradients and additional flow compensation (Figure 1A). A reduced FOV was obtained using outer volume suppression. The diffusion weighting gradients were applied in 3 orthogonal directions with for b-values of 30,60 , $90,120 \mathrm{~s} / \mathrm{mm}^{2}$ and in 12 directions for a b-value of 300 $\mathrm{s} / \mathrm{mm}^{2}$. Additionally 4 non-weighted images were acquired resulting in 28 volumes. Every volumes was acquired twice resulting in a total acquisition time of $15 \mathrm{~min}$ for a heart rate of $60 \mathrm{bpm}$. Further parameters were; FOV: $280 \times 150 \mathrm{~mm}^{2}$, voxel size: $6 \times 2.5 \times$ $2.5 \mathrm{~mm}^{3}$, slices: 16 , BW-EPI: $42 \mathrm{~Hz}$ TR: 8 heartbeats,
TE: 55 ms. First data was registered to correct for heartand breathing motion using a 2D non-rigid method followed by Rician noise suppression. Finally data was fitted to: $\mathrm{S}(\mathrm{b}, \mathrm{g})=\mathrm{S} 0\left((1-\mathrm{fr}) \exp \left(-\mathrm{b} g \mathbf{D ~ g}^{\mathrm{T}}\right)+\right.$ fr $\exp (-\mathrm{b} \mathrm{g}$ D $\left.\left.\mathrm{g}^{\mathrm{T}} \mathrm{D}^{*}\right)\right)$ using a constrained non-linear least squares method. Fiber tractography was performed the vIST/e toolbox with a step size of 0.2 voxel. Stopping criteria were $0.1<\mathrm{FA}<0.6$ and an angle change of $20^{\circ}$ per step.

\section{Results}

The corrected DWI images for $\mathrm{b}=300 \mathrm{~s} / \mathrm{mm}^{2}$ are shown in Figure 1B. Figure 2A to 2D show parameter maps for $\mathrm{MD}, \mathrm{FA}, \mathrm{f}$ and $\mathrm{D}^{*}$ resulting from the combined IVIM and tensor fit. The average values for the whole heart were $1.67 \pm 0.49^{*} 10^{-3} \mathrm{~mm}^{2} / \mathrm{s}, 0.46 \pm 0.20,0.27 \pm$ $0.16,52.68 \pm 52.61 * 10^{-3} \mathrm{~mm}^{2} / \mathrm{s}$ respectively. The cardiac helical fiber organization could be reproduced by fiber tractography as shown in Figure 2E to $2 \mathrm{G}$ where the fiber tracts are color coded for the helix angle.

\section{Conclusions}

In this study we have shown that it is feasible to acquire whole heart DTI and IVIM data within a 15 min protocol in free breathing. Using this approach we were able to quantify the diffusion and perfusion and visualize the fiber architecture.

\section{Funding}

Not applicable. 

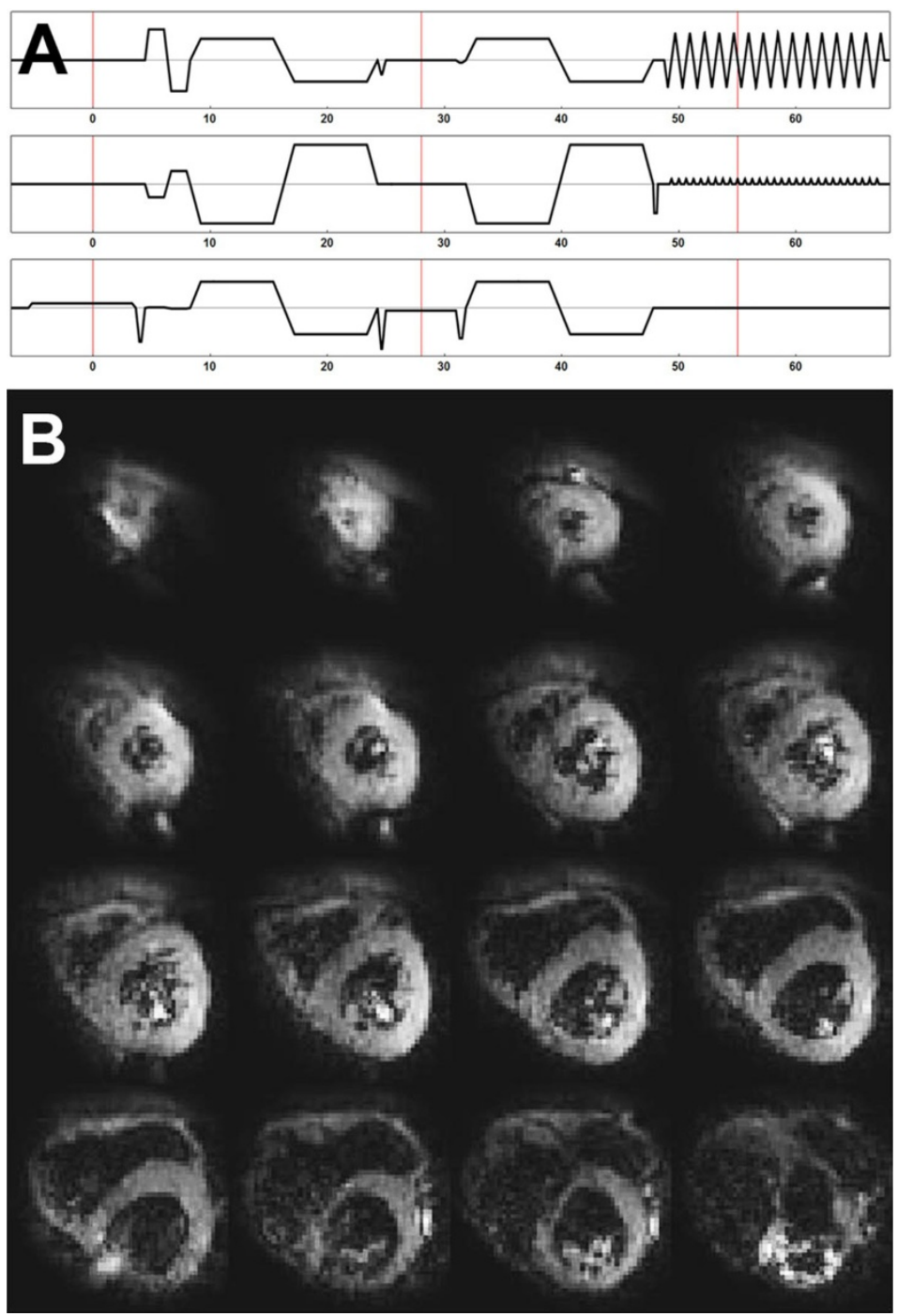

Figure 1 A) Diffusion-weighted SE sequence with bipolar diffusion encoding and flow compensation gradients directly after the 90 degree slice selection. B) The acquired single shot diffusion weighted data for $\mathrm{b}=300 \mathrm{~s} / \mathrm{mm}^{2}$, with a voxel size of $6 \times 2.5 \times 2.5 \mathrm{~mm}$ and TE $=55 \mathrm{~ms}$
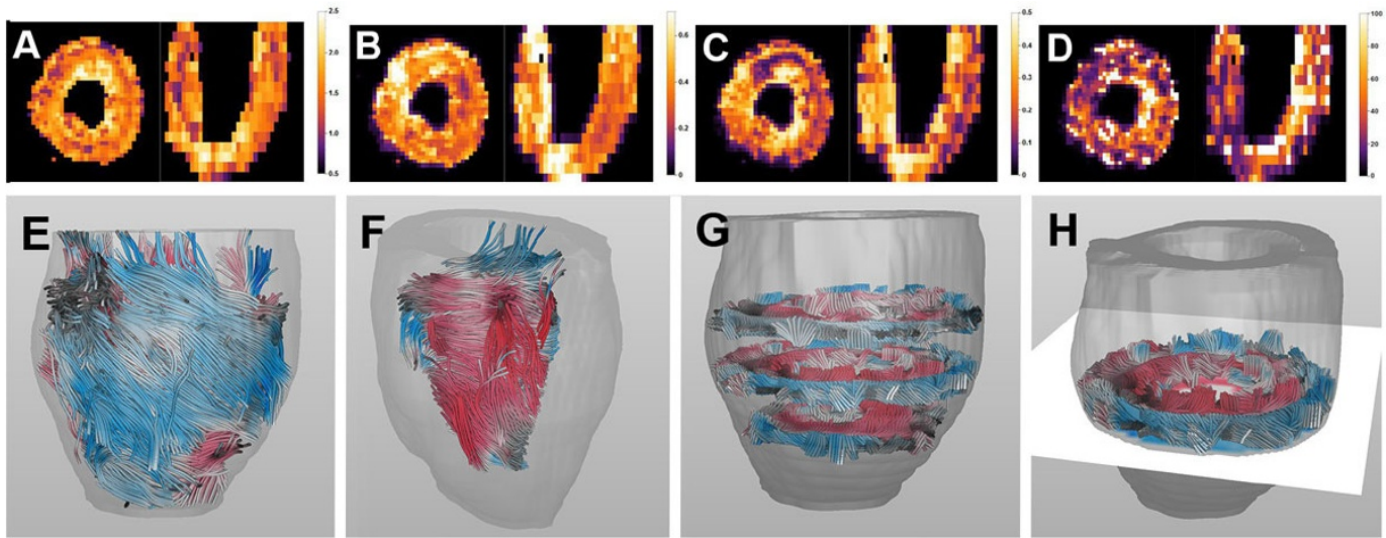

Figure 2 A-D) Parameter maps based on the IVIM fit (A: MD in 10-3 mm²/s, B: FA, C: fraction, D: $D^{*}$ in in $\left.10-3 \mathrm{~mm}^{2} / \mathrm{s}\right)$. E-F) whole heart fiber tractography based on the IVIM tensor fit color coded for helix angle. (E: whole heart, F: Inside of the myocardial wall with papillary muscle, G-H: local fiber orientation for different cross sections) 


\section{Authors' details}

'Department of Radiology, University Medical Center, Utrecht, Netherlands.

${ }^{2}$ Department of Biomedical Engineering, Biomedical NMR, Eindhoven

University of Technology, Eindhoven, Netherlands. ${ }^{3}$ Department of

Radiology, Academic Medical Center, Amsterdam, Netherlands. ${ }^{4}$ Department

of Cardiology, Division Heart \& Lung, University Medical Center, Utrecht,

Netherlands.

Published: 16 January 2014

doi:10.1186/1532-429X-16-S1-015

Cite this article as: Froeling et al: Feasibility of in vivo whole heart DTI

and IVIM with a 15 minute acquisition protocol. Journal of Cardiovascular

Magnetic Resonance 2014 16(Suppl 1):O15.

Submit your next manuscript to BioMed Central and take full advantage of:

- Convenient online submission

- Thorough peer review

- No space constraints or color figure charges

- Immediate publication on acceptance

- Inclusion in PubMed, CAS, Scopus and Google Scholar

- Research which is freely available for redistribution

Submit your manuscript at www.biomedcentral.com/submit 